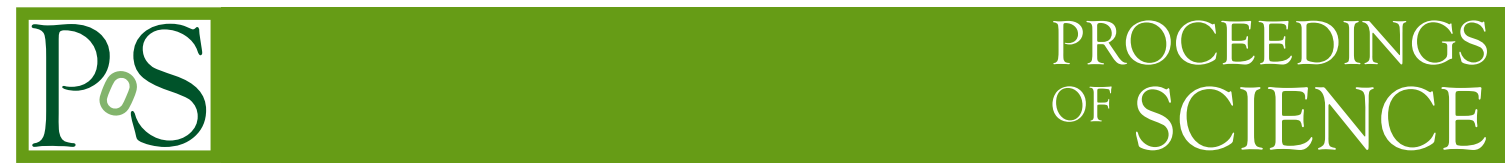

\title{
Models for Microquasars
}

\author{
Julien Malzac* \\ CESR, OMP, UPS, CNRS; Toulouse, France \\ E-mail: malzac@cesr.fr
}

I review current models used to interpret the spectra and variability of microquasars. Among other things, I discuss the structure of the accretion flow and its dependence on mass accretion rate, the intrinsic connection between hot comptonizing corona and compact radio jet in the hard state, as well as possible models for the spectral hysteresis observed during outbursts of transient sources. Finally I comment on several models for the non-poissonian X-ray noise in black hole binaries which, at least in some instances, is suspected to be associated with some form of coupling between disc and jet activity.

VI Microquasar Workshop: Microquasars and Beyond September 18-22, 2006

Como, Italy

${ }^{*}$ Speaker. 


\section{X-ray spectral states and the structure of the accretion flow}

Most of the luminosity of accreting black holes is emitted in the X-ray band. This X-ray emission is strongly variable. A same source can be observed with very different X-ray spectra (see e.g. [1]). Fig. 1 shows various spectra from the prototypical source Cygnus X-1 observed at different epochs. There are two main spectral states that are fairly steady and most frequently observed. At high luminosities the accretion flow is in the High Soft State (HSS), caracterized by a strong thermal disc and reflection components (believed to be due to X-ray illumination of the accretion disc) and a weak and non-thermal component believed to be produced through nonthermal (or hybrid thermal/non-thermal) comptonisation in a hot corona. At luminosities lower than a few percent of Eddington $\left(L_{E d d}\right)$, the sources are generally found in the Low Hard State (LHS) in which the disc blackbody and reflection features are much weaker, while the corona has a thermal distribution of comptonising electrons and dominates the luminosity output of the system. Beside the LHS and HSS, there are several other spectral states that often appear, but not always, when the source is about to switch from one of the two main states to the other. Those states are more complex and difficult to define. We refer the reader to [6] and [7] for two different spectral classifications based on X-ray temporal as well as spectral criteria and radio emission [8]. In general, their spectral properties are intermediate between those of the LHS and HSS.

The different spectral states are usually understood in terms of changes in the geometry of the accretion flow. In the HSS, according to the standard scenario sketched in Fig. 1, a standard geometrically thin disc extends down to the last stable orbit and is responsible for the dominant thermal emission. The non-thermal hard X-ray emission is generally believed to originate from an Accretion Disc Corona (ADC) constituted of small active regions located above and below the disc. Due to magnetic buoyancy, the magnetic field lines rise above the accretion disc, transporting a significant fraction of the accretion power into the corona where it is then dissipated through magnetic reconnection [9]. This leads to particle acceleration in the ADC. A population of high energy electrons is formed which then cools down by up-scattering the soft photons emitted by the disc. This produces the high energy non-thermal emission which in turn illuminates the disc forming strong reflection features (see e.g. [1]). There are no strong alternatives to ADC model for the soft state. The most relevant is probably the bulk motion model [10] which attributes the hard Xray emission to Comptonisation in the innermost region by the plasma inflowing into the black hole. However, as pointed out in [11], this model predicts a sharp cut-off in the high energy spectrum around $100 \mathrm{keV}$, and therefore does not explain the MeV emission observed in several HSS sources. In the LHS, the standard geometrically thin disc does not extend to the last stable orbit, instead, the weakness of the thermal features suggests that it is truncated at distances ranging from a few hundreds to a few thousands gravitational radii from the black hole (typically 1000-10000 km). In the inner parts, the accretion flow takes the form of a hot geometrically thick, optically thin disc. A solution of such hot accretion flows was first proposed by Shapiro et al [12]. In these hot acccretion flows the gravitational energy is converted in the process of viscous dissipation into the thermal energy of ions. The main coupling between the electrons and the ions is Coulomb collision which is rather weak in the hot thin plasma. Since radiative cooling of the ions is much longer than that of the electrons, the ions temperature is much higher than the electron temperature. This two temperature plasma solution is thermally unstable [13] but can be stabilized if advection of the hot 
gas into the black hole dominates the energy transfert for ions as in advection dominated accretion flows (ADAF) solutions (see [14], [15], [16]). Emphazising the fact that in the ADAF solution the accreting gas has a positive Bernouilli parameter, Blandford and Begelman [17] proposed a variant of the ADAF model in which a significant fraction of the accreting material is advected into an outflow rather than in the black hole (ADIOS). In these hot accretion flow solutions most of the accretion power is either swallowed by the black hole or converted into kinetic power of an outflow. They are therefore radiatively inefficient accretion flows (RIAF). Yuan [18] has shown that a hot flow may also exist in the higher accretion rate regime where the coupling between electrons and ions becomes effective: the flow is then radiatively efficient. In all these hot flow models the electrons have a thermal distribution and cool down by Comptonisation of the soft photons coming from the external geometrically thin disc, as well as IR-optical photons internally generated through self-absorbed synchrotron radiation. The balance between heating and cooling determines the electron temperature which is found to be of the order of $10^{9} \mathrm{~K}$, as required to fit the spectra. The weak reflection features of the LHS are produced through illumination of the cold outer disc by the central source. By extension, the hot accretion flow of the LHS is frequently refered to as "corona" despite the lack of a direct physical analogy with the rarefied gaseous envelope of the sun and other stars or the ADC of the HSS.

\section{An alternative model for the LHS: accretion disc corona}

The presence of the hot accretion disc is not the only possibility to explain the LHS. It was suggested long ago that instead, a real ADC system similar to that of the HSS but with a pure Maxwellian distribution of comptonising electrons, may reproduce the spectra as well [19] [20]. This would imply a cold geometrically thin disc extending down very close to the black hole in the LHS. Unlike in the HSS, this disc does not produce a strong thermal component in the Xray spectrum because it is too cold. Indeed, it has a much lower temperature because most of the accretion power is not dissipated in the disc, instead, it is assumed to be transported away to power a strong ADC and the compact jet. This, at least in principle, could be achieved either through transport via buyonancy of the magnetic field [21][22] or through the torque exerted on the accretion disc by a strong magnetic field threading the disc and driving the jet [23].

Due to the complexity of the ADC physics it is not possible to obtain simple analytical solutions predicting the main properties of the ADC unless some parametrization of the energy transfert between the disc and the corona is used (see e.g. [24]). However, in practice, the appearance of the ADC does not depend on the details of the energy transport and dissipation mechanisms. Indeed, Haard and Maraschi [25] pointed out the existence of a strong radiative feedback between the cold disc and the hot corona. A fixed fraction of the power dissipated in the corona in the form of hard $\mathrm{X}$-ray photons intercepts the cold disc where it is absorbed. As a consequence the disc heats up, and the absorbed energy is finally reemitted in the form of thermal soft photons. A fraction of those soft photons re-enters the corona providing the major cooling effect to the corona trough inverse Compton. Due to this strong feedback from the disc, the cooling rate of the corona scales like the heating rate. The coronal temperature is determined only by the geometry of the ADC (that controls the fraction of coronal power that returns to the corona in the form of soft photons). For instance, an extended ADC sandwiching the cold accretion disc, would intercept more cooling pho- 

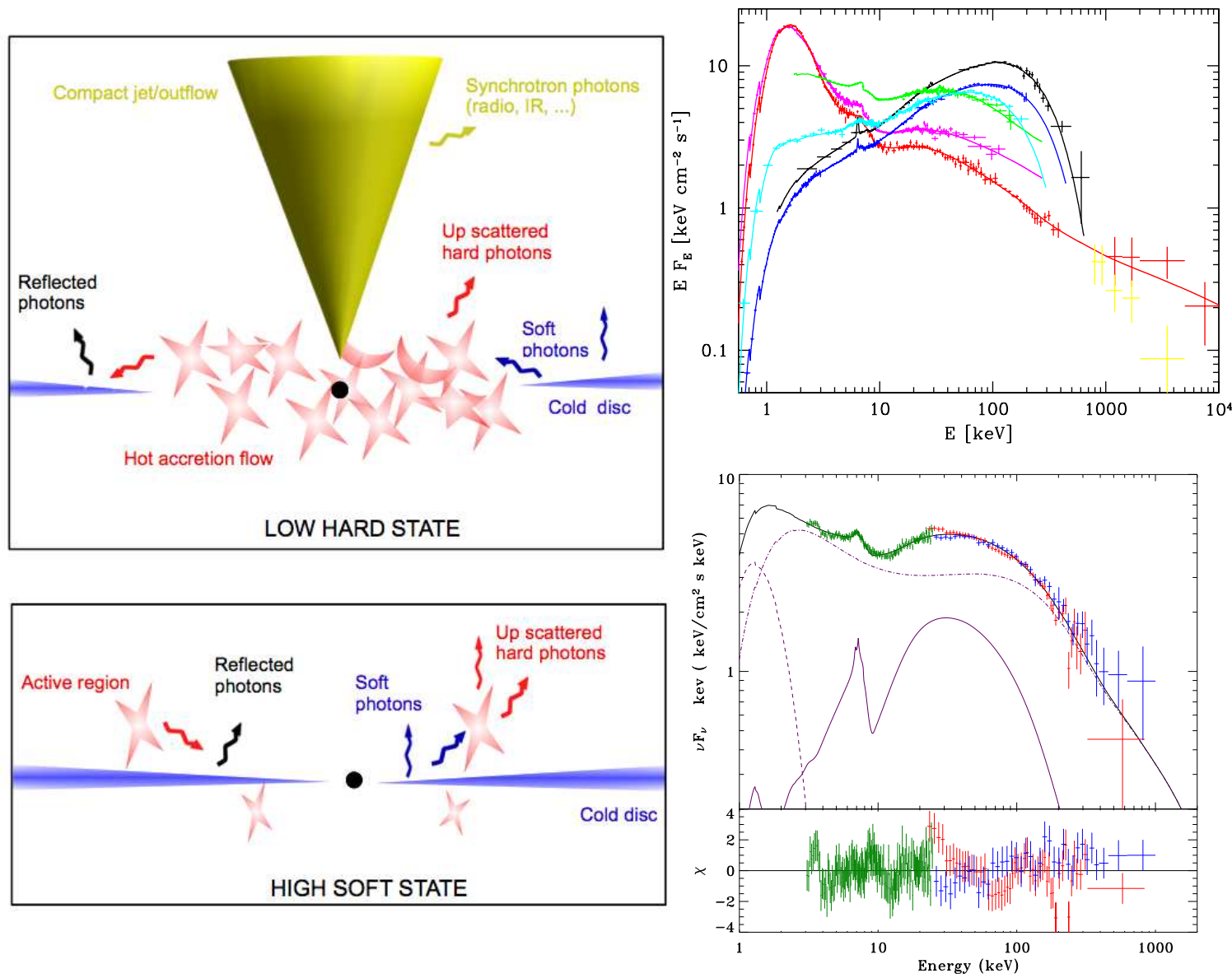

Figure 1: Left hand side panels: Structure of the accretion flow during LHS (top) and HSS (bottom) according to the standard scenario. Right hand side panels: Observed spectra of Cygnus X-1 . Upper panel (from [2]): the LHS (black and blue), HSS (red, magenta), IMS (green, cyan). The solid curves give the bestfit Comptonization models (thermal in the hard state, and hybrid, thermal-nonthermal, in the other states). Lower panel: Time averaged INTEGRAL spectrum of Cygnus X-1 during a mini-state transition (intermediate state). The data are fitted with the thermal/non-thermal hybrid Comptonisation model EQPAIR with mono-energetic injection of relativistic electrons. The lighter curves show the reflection component (solid), the disc thermal emission (dashed) and the Comptonised emission (dot-dashed). The green, red and blue crosses show the JEM-X, IBIS/ISGRI and SPI data respectively. See [3] for details.

tons from the accretion disc than a patchy corona made of a few compact active regions covering a small fraction of the disc, and therefore would have a lower temperature and a softer comptonised X-ray spectrum. During the nineties several groups [26] [27][28] performed detailed computations of the resulting equilibrium spectra for various geometries. They concluded that the ADC has to be patchy in order to produce spectra that are hard enough [29][30] even when the effects of ionisation on the disc albedo are accounted for [31]. Yet, those ADC models had an important problem: the production of an unobserved strong thermal component due to reprocessing of the radiation illuminating the disc. Indeed, in the case of an active region emitting isotropically, about half of the luminosity intercepts the disc and the thermal reprocessing component is comparable in luminosity to the primary emission. Actually, in observed LHS spectra this thermal disc component is so weak 

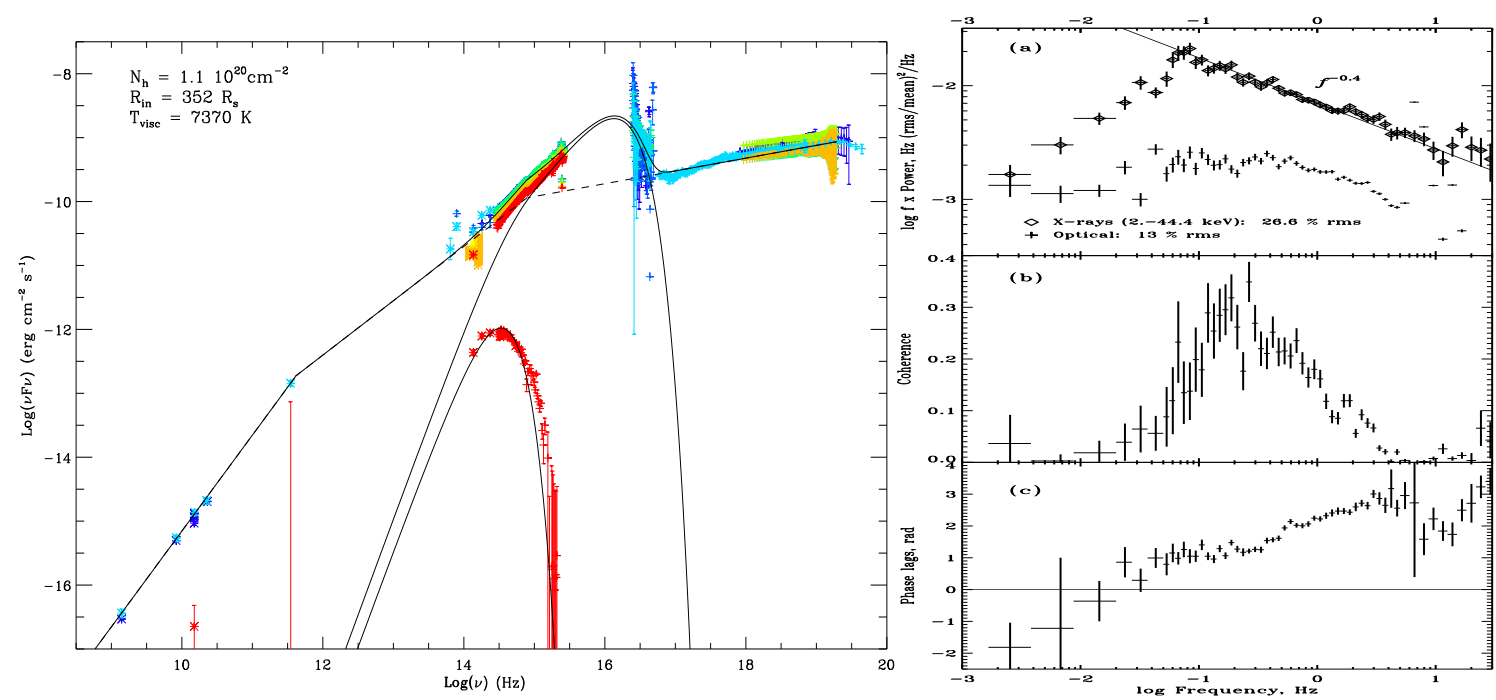

Figure 2: Left: Spectral Energy Distribution of XTE J1118+480 during its 2000 outburst (from [4]). Right: The optical/X-ray correlations of XTE J1118+480 in the Fourier domain. a) X-ray and optical power spectra. The counting noise was subtracted. b) X-ray/optical coherence. c) phase-lags as function of Fourier frequency. A positive lag implies that the optical is delayed with respect to the X-rays (from [5]).

that it is barely detectable. Morevover, the amplitude of the reflection features observed in the LHS are lower than what is expected from an istotropic corona by at least a factor of 3 , in some cases they are so weak that they are not even detected. This led to consider models where the coronal emission is not isotropic. It was suggested that the corona is unlikely to stay at rest with respect to the accretion disc [32]. Due to the anisotropy of the dissipation process or simply due to radiation pressure from the disc, the hot plasma is likely to be moving at mildly relativistic velocities. Then, due to Doppler effects, the X-ray emission is strongly beamed in the direction of the plasma velocity. In the case of a velocity directed away from the accretion disc (outflowing corona), the reprocessing features (both reflection and thermalised radiation) are strongly suppressed. Moreover, due to the reduced feedback from the disc, the corona is hotter, and harder spectra can be produced. Detailled non-linear Monte-Carlo simulations of this dynamic ADC equilibrium are presented in [33] . A comparison of these results with observations showed that compact active regions of aspect ratio of order unity, outflowing with a velocity of 30 percent of the speed of light could reproduce the LHS spectrum of Cygnus X-1. Moreover, since the velocity of the coronal plasma controls both the strength of the reflection features and the feedback of soft cooling photons from the disc, it predicts a correlation between the slope of the hard X-ray spectrum and the amplitude of the reflection component. Such a correlation is indeed observed in several sources [34] and is well matched by this model. Recently the ADC models for the LHS obtained some observationnal support, with the discovery of a relativistically broadened iron line in the LHS of GX339-4 [35]. Such relativistically broadened lines require disc illumination taking place very close to the black hole. This observation suggests that, at least in some cases, a thin disc is present at, or close to, the last stable orbit in the LHS. Incidentally, in the framework of the outflowing ADC model, the velocity of the corona required to fit hard state spectra appears to be comparable to the estimates of the compact radio jet velocity [36]. This suggests a direct connection between corona and jet. 


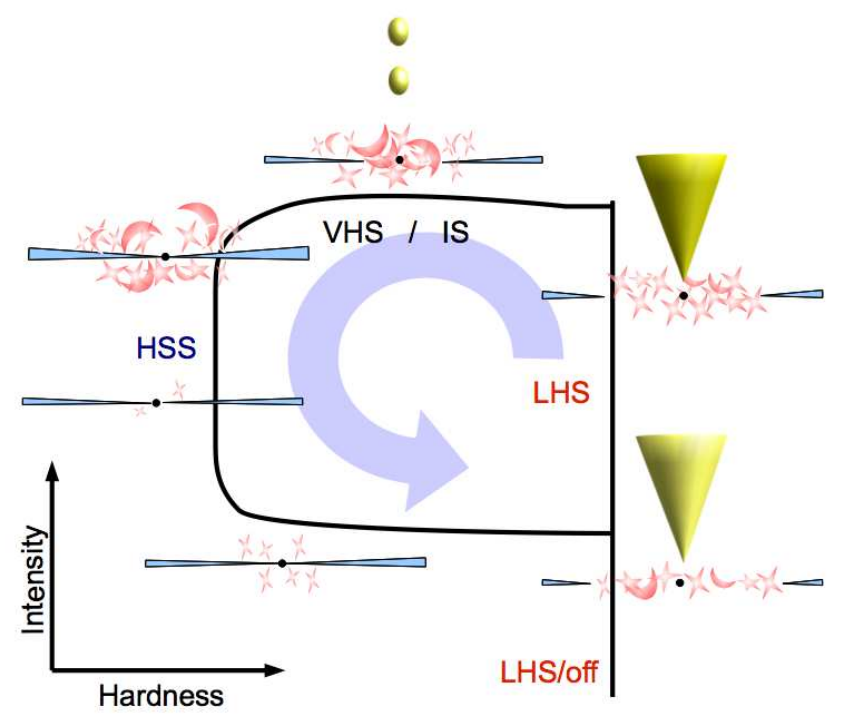

Figure 3: The black curve shows the typical path followed by a black hole X-ray transient in the hardness vs intensity diagram during an outburst. The various sketches illustrate the standard scenario for the evolution of the geometry of the corona-disc-jet system along this path (see text).

\section{The jet corona connection}

Multi-wavelength observations of accreting black holes in the LHS have shown the presence of an ubiquitous flat-spectrum radio emission (see e.g [8]), that may extend up to infrared and optical wavelengths (see Fig. 2). The properties of the radio emission indicate it is likely produced by synchrotron emission from relativistic electrons in compact, self-absorbed jets [37] [38]. This idea was confirmed by the discovery of a continuous and steady milliarcsecond compact jet around Cygnus X-1 [39]. Moreover, in LHS sources a tight correlation has been found between the hard $\mathrm{X}$-ray and radio luminosities, holding over more than three decades in luminosity [36]. In contrast, during HSS episodes the sources appear to be radio weak [40], suggesting that the Comptonising medium of the low/hard state is closely linked to the continuous ejection of matter in the form of a small scale jet. When the importance of the connection between radio and X-ray emission was realised, it was proposed that the hard X-ray emission could be in fact synchrotron emission in the jet, rather than comptonisation in a hot accretion flow/corona [41]. However, it seems that in most sources the synchrotron emission alone is not enough to reproduce the details of the X-ray spectra. In the most recent version of this model a thermal Comptonisation component was added which appears to provide a dominant contribution to the hard X-ray spectrum [42]. This component is supposedly located at the base of the jet which forms a hot plasma very similar to an ADC.

In the context of $\mathrm{ADC} / \mathrm{hot}$ disc models the correlation between $\mathrm{X}$-ray and radio emissions, simply tells us that the corona and the compact jet of the LHS are intimately connected. A strong corona may be necessary to launch a jet and/or could be the physical location where the jet is accelerated or launched [22]. Jet spectral components have recently been added to the hot disc models and make it possible to produce good fits of the whole spectral energy distribution from radio to hard X-rays [43], although the physics of the jet/ADAF connection has not been worked out yet. 
Following a different approach, Körding et al. [44] show that the observed X-radio correlation can be reproduced provided that the hot flow/corona is radiatively inefficient (i.e. luminosity scales like the square of mass accretion rate) and that a constant fraction of the accretion power goes into the jet (i.e. jet power scales like mass accretion rate). However there is presently no detailed model to explain how the physical connection between jet and corona operates. A possible basic explanation was proposed by Meier [45] for ADAF like accretion flows and later extended to the case of ADC by Merloni and Fabian [22]. It goes as follows. Models and simulations of jet production indicate that jets are driven by the poloidal component of the magnetic field [47][46][23]. If we assume that the magnetic field is generated by dynamo processes in the disc/corona, the strengh of the poloidal component is limited by the scale height of the flow [48][45][22]. Therefore geometrically thick accretion flows should be naturally more efficient at launching jets.

Despite this strong link between the corona and outflow in the LHS, there are indications that in Intermediate States (IS) the jet is connected to the accretion disc rather than the corona. For instance, Malzac et al. [3] report the results of an observation of Cygnus X-1 during a mini-state transition (see Fig. 1). The data indicate that the radio jet luminosity is anti-correlated with the disc luminosity and unrelated to the coronal power. This is in sharp contrast with previous results obtained for the LHS, and suggests a different mode of coupling between the jet, the cold disc, and the corona in Intermediate States. The reason for the anti-correlation is most probably that state transitions are associated with a redistribution of the available accretion power between the compact jet and the cold accretion disc. In the standard scenario described in section 1, this redistribution of accretion power could occur because the jet shrinks as the inner radius of the outer disc moves closer to the black hole.

\section{Evolution of the geometry during outburst: the hysteresis problem}

Transient black hole binaries are a class of X-ray binaries that are detected only occasionally when they show some period of intense activity (outbursts) lasting for a few months (see e.g. [49]). These outbursts have recurrence times ranging from years to dozens of years. Between two episodes of activity the source is in an extremely faint quiescent state. During an outburst, the source luminosity varies by many orders of magnitude. The study of the spectral evolution during outbursts is perfectly suited to study the dependence of the structure of the accretion flow on the mass accretion rate. This spectral evolution is conveniently described using hardness intensity diagrams. Those diagrams show the evolution of the source luminosity as a function of spectral hardness (defined as the ratio of the observed count rates in a high to a lower energy band). Typically, during outbursts the sources follow a $q$-shaped path in the hardness intensity diagram (see Fig. 3). Initially in quiescence with a very low luminosity, they are in the LHS with a high hardness. In the standard scenario ${ }^{1}$, the cold disc is truncated very far away from the black hole, and the emission is dominated by the hot accretion flow. Then, as luminosity increases by several orders of magnitude the hardness remains constant. The structure of the accretion flow is stable, the cold disc inner radius probably moving slowly inward. During this rise in X-ray luminosity

\footnotetext{
${ }^{1}$ We note that a different geometrical interpretation of the outburts cycle in terms of a jet dominated accretion flow was recently proposed by Ferreira et al. [50].
} 
a compact jet is present and its radio emission correlates with the X-ray emission. At a luminosity that is always above a few percent of Eddington, the inner radius of the cold accretion disk decreases quickly. This reduction of the inner disc radius is associated with either the cold disk penetrating inside the hot inner flow, or the later collapsing into an optically thick accretion disk with small active regions of hot plasma on top of it ([2]). In both cases the enhanced soft photon flux from the disk tends to cool down the hot phase, leading to softer spectra. The source therefore moves from the right toward the left hand side at almost constant luminosity. During this hard to soft state transition the sources usually show a strong coronal activity (both the disc and corona are strong), this is also during this transition that optically thin radio flares associated with relativistic and sometimes superluminal ejections are observed. This region of the hardness intensity diagram is often called the Very High State (VHS). Once a source has reached the upper left hand corner of the hardness intensity diagram, it never turns back to the LHS following the same path. Instead when the luminosity decreases, it stays in the HSS and goes down vertically and the strength of the non-thermal corona decreases as well. This goes on until the luminosity reaches $\simeq 0.02$ percent of Eddington. Then it moves horizontally to the right, back to the LHS, and then down vertically to quiescence.

It is to be noted that the luminosity of state transition from soft to hard seems to be relatively fixed around 0.02 percent of Eddington [51]. On the other hand the hard to soft transition can vary, even for the same source, by several orders of magnitude and it is always higher than the soft to hard transition luminosity. The question of what controls the different transitions luminosity is still open. Indeed, such a complex behaviour is not expected a priori. In all accretion models, there is only one relevant externally imposed parameter: the mass acretion rate. It was not anticipated that for the same luminosity the accretion flow could be in different states. It was recently suggested that this complex behaviour could be explained by the existence of a second parameter that could be the magnetic flux advected with the accreting material [52]. But the most detailed explanations so far are based on the idea that this second parameter is nothing else than the history of the system. In other word the accretion flow would present an hysteresis behaviour because going from LHS to HSS is not the same thing as going from HSS to LHS. Within this line of reasoning, a promising idea was proposed by Meyer-Hofmeister et al [53] who suggested that the hysteresis could be linked to a condensation/evaporation equilibrium in an ADC system. They assume an advective corona on top of a thin optically thick accretion disc and allowing for mass exchange between the corona and the disc. Depending on the temperature and density in the corona and the disc, the material in the corona may condensate into the disc and the disc may evaporate. Meyer-Hofmeister et al [53] have computed the resulting equilibrium between an accretion disc and a corona as a function of the distance to the black hole and mass accretion rate (see also [54] [55]). They have found that at large distance the conditions are such that an equilibrium solution can always be found. At closer distances from the black hole, disc evaporation becomes more and more important and, under some circumstances there is a critical radius below which the disc fully evaporate and no thin disc is possible. Below this radius we are then left with a pure advection dominated hot accretion flow. The location of this transisition radius depends on the mass accretion rate. At low mass accretion rate the transition radius is at $10^{3}$ to $10^{4}$ Schwarschild radii and decreases slowly as the accretion rate increases until the critical mass accretion of about a few percent of Eddington is reached. Then, the inner radius suddenly drops to the last stable orbit: this is the hard to soft state 
transition. As long as the source is in the LHS the flux of soft cooling photons in the corona is weak, and, as a consequence the corona is hot and evaporation dominates over condensation. Once the HSS is reached however, there is a disc down to the last stable orbit emitting a strong thermal soft emission that efficiently cools the corona, keeping the condensation process strong even when the mass accretion rate decreases. As a consequence the transition from the soft to LHS occurs at a luminosity that is about one order of magnitude lower than the hard to soft transition.

Although this model so far provides the only quantitative explanation of the hysteresis behaviour, it is not perfect. First, the presence of the jet, possibly impacting on the disc/corona equilibrium is completely ignored. Second, this model does not explain why the transition LHS to HSS can occur at different luminosities (the transition luminosity is quite constrained), nor the absence of hysteresis behaviour in persistent sources such as Cygnus X-1. A second independent parameter may still be required.

\section{Timing properties}

$\mathrm{X}$-ray binaries harbour a strong rapid X-ray variability which presents a very complex and richly documented phenomenology [56]. Modeling this fast variability is very challenging. The main problem that any model must overcome is a time-scale problem. Indeed, most of the high energy photons (and variability) must originate deep in the potential. The variability is therefore expected to occur on time-scales of the order of the dynamical time-scale close to the black hole, which is of order of $1 \mathrm{~ms}$ for a ten solar mass black hole. On the other hand, observations show that most of the X-ray variability occurs on time-scales ranging from 0.1 to $10 \mathrm{~s}$ (see e.g. panel $a$ on the right hand side of Fig. 2). In comparison there is virtually no variability on time-scales shorter than 0.01 s. Thinking in terms of Power Density Spectrum (PDS), the observed power requires short distances from the black hole, but the observed frequencies imply large distances.

Another intriguing feature of the rapid variability of black hole binaries is the existence of delays between energy bands (the hard photons lagging behind the softer photons [57]). These timelags are often believed to be associated to the time required for a photon to gain energy through multiple compton scaterring as it travels in the corona [58]. But this explanation is probably wrong: First, the observed time-lags are very long and would require an unplausibly large Comptonizing corona $(\sim 10000 \mathrm{Rg})$. But more fundamentally, the time-lags cannot be due to Comptonisation delays because this requires the variability to be driven by fluctuations of the soft seed photon luminosity while the hot corona keeps constant physical parameters. Indeed, Malzac and Jourdain [59] have shown that in a real situation the corona responds to the fluctuations of the soft photon field and damps out any variability. For example, if the soft photon density is suddenly increased while the dissipation rate in the corona is kept constant, then the temperature drops almost instantaneously (on a Compton cooling time scale which is much shorter than the light crossing time). In a fluctuating soft photon field, the coronal temperature tends to adjust very quickly to keep the luminosity constant. Models in which the variability is driven by the soft photon field might be successful in reproducing the shape of the power spectra or time-lags (see e.g. [60]) but they cannot produce the strong variability rms amplitudes of 10 to 40 percent that are typically observed. Therefore the rapid variability must be driven by changes in the physical parameters of the corona (most likely the hot plasma heating rate), rather than by fluctuations of the soft seed photon input. 
There are several models that successfully reproduce the PSD shapes and amplitude as well as other observed properties such as the time-lags. So-called 'shot noise models' (see e.g. [61]) attempt to phenomenologically describe the light curves as a succession of random flares with specific time scales, amplitudes, and occurence rates. More physically motivated models may involve turbulence in accretion discs [62], avalanche of magnetic flares [63], or MHD in the plunging region [64]. Sometimes, the time scale problem is solved by invoking a mechanism correlating short time-scale fluctuations to generate the observed long time-scale fluctuations. However it was shown by [65] that the variability is non-linear. Indeed, they demonstrated the existence of a linear correlation between the measured count rate and the rms variability that seems to be present in all sources and in all spectral states and on all time-scales. This correlation shows that the short timescales are modulated by the longer time scales. This appears to favour models where the variability is built from large to short times-scales.

A possibility could be that fluctuations are generated at large distance and propagate inward. For instance, Lyubarskii [66] postulates fluctuations in viscosity on local inflow timescale (itself viscous) over a wide range of distances $r$ from the black hole and computes the resulting fluctuations of the mass accretion rate at the inner disc radius. For fluctuations independent of $r$, this gives a power spectrum $p(f) \propto 1 / f$ i.e. slow variations of large amplitude. Kotov et al. [67] improved upon this model and showed that assuming a harder spectrum close to the black hole produces a logarithmic dependence of time-lags on energy, as observed. The original model by Lyubarskii had no physical description for the viscous fluctuations. A likely mechanism could be magnetic dynamo in the disc. However, dynamo processes vary on a dynamical time-scale i.e. much faster than the viscous time fluctuations assumed by Lyubarskii. In a similar framework, King et al. [68] suggested that mass accretion rate fluctuations on long time scales could be produced if independent dynamos at a range of disc annuli can 'cooperate'. At each radius a dynamo produces a magnetic field fluctuating on a dynamical time-scale, both in amplitude and orientation. Very occasionally the magnetic field is aligned over a large surface of the disc. This allows the generation of a local wind or outflow, also causing rapid angular momentum loss in the disc and ultimately a temporary increase of the mass accretion rate in the inner pat of the disc leading to a X-ray flare occuring on time-scales $>t_{d y n}$. King et al. [68] and then Mayer and Pringle [69] showed that this kind of models may reproduce observed power spectra. The current version of the model assumes that the variability is generated in a geometrically thin disc extending close to the black hole. Actually what is observed is the X-ray variability of the corona (either ADC or RIAF). The model does not explain how the fluctuation of physical parameters in the thin disc affects those in the corona. Moreover, observations in the soft state have shown that despite the strong coronal activity, the disc thermal emission is extremely stable. Most of the variability appears to be generated in the corona rather than in the thin disc [70].

Despite these problems, an interesting feature of this model is that it connects the X-ray timing properties with the formation of outflows, basically predicting a correlation between rapid jet and $\mathrm{X}$-ray activity. Of course variability of the radio jet on time-scale as short as seconds or less is impossible to observe with present radio instrumentation. However, in some sources, such as XTE $\mathrm{J} 1118+480$, the jet has a significant contribution in infrared and optical, making fast jet photometry possible. In XTE J1118+480, fast optical and UV photometry has revealed a rapid optical/UV flickering presenting complex correlations with the X-ray variability [71] (see Fig. 2). This corre- 
lated variability cannot be caused by reprocessing of the X-rays in the external parts of the disc. Indeed, the optical flickering occurs on average on shorter time-scales than the X-ray one [71], and reprocessing models fail to fit the complicated shape of the X-ray/optical cross correlation function. Spectrally, the jet emission seems to extend at least up to the optical band (see Fig. 2 and [4]), although the external parts of the disc may provide an important contribution to the observed flux at such wavelengths. The jet activity is thus the most likely explanation for the rapid observed optical flickering. For this reason, the properties of the optical/X-ray correlation in XTE J1118+480 might be of primary importance for the understanding of the jet-corona coupling and the ejection process. One of the most intriguing of these properties is that the two lightcurves seem to be related trough a differential relation. Namely, if the optical variability is representative of fluctuations in the jet power output $P_{\mathrm{j}}$, the data suggest that the jet power scales roughly like $P_{\mathrm{j}} \propto-\frac{d P_{\mathrm{x}}}{d t}$, where $P_{\mathrm{x}}$ is the X-ray power [5]. Such a relationship is not expected in the model by King et al. [68]. On the other hand, Malzac et al [72] have shown that the complex X-ray/optical correlations could be understood in terms of an energy reservoir model. In this picture, it is assumed that large amounts of accretion power are stored in the accretion flow before being channelled either into the jet or into particle acceleration/ heating in the Comptonizing region responsible for the X-rays. The optical variability is produced mainly from synchrotron emission in the inner part of the jet at distances of a few thousands gravitational radii from the hole. It is assumed that at any time the optical flux $O_{p t}$ (resp. X-ray flux) scales like the jet power $P_{\mathrm{j}}$ ( plasma heating power $P_{\mathrm{x}}$ ).

The essence of the model can be understood using a simple analogue: Consider a tall water tank with an input pipe and two output pipes, one of which is much smaller than the other. The larger output pipe has a tap on it. The flow in the input pipe represents the power injected in the reservoir $P_{\mathrm{i}}$, that in the small output pipe the $\mathrm{X}$-ray power $P_{\mathrm{x}}$ and in the large output pipe the jet power $P_{\mathrm{j}}$. If the system is left alone the water level rises until the pressure causes $P_{\mathrm{i}}=P_{\mathrm{j}}+P_{\mathrm{x}}$. Now consider what happens when the tap is opened more, causing $P_{\mathrm{j}}$ to rise. The water level and pressure (proportional to $E$ ) drop causing $P_{\mathrm{x}}$ to reduce. If the tap is then partly closed, the water level rises, $P_{\mathrm{j}}$ decreases and $P_{\mathrm{x}}$ increases. The rate $P_{\mathrm{x}}$ depends upon the past history, or integral of $P_{\mathrm{j}}$. Identifying the optical flux as a marker of $P_{\mathrm{j}}$ and the $\mathrm{X}$-ray flux as a marker of $P_{\mathrm{x}}$ we obtain the basic behaviour seen in XTE J1118+480. In the real situation, it is envisaged that the variations in the tap are stochastically controlled by a shot noise process. There are also stochastically-controlled taps on the input and other output pipes as well. The overall behaviour is therefore complex.

This simple model is largely independent of the physical nature of the energy reservoir. In a real accretion flow, the reservoir could take the form of either electromagnetic energy stored in the X-ray emitting region, or thermal (hot protons) or turbulent motions. The material in the disc could also constitute a reservoir of gravitational or rotational energy behaving as described above. A strong requirement of the model is that the jet power should be at least a few times larger than the X-ray power. This fact seems to be independently supported by recent results on the study of the X-ray radio correlation in black holes and neutron stars indicating that for black holes in the LHS the jet power dominates over the X-ray emission [44]. Finally this model accounts for the non-linearity: most of the $\mathrm{X}$-ray noise is due to slow fluctuations of the outflow dissipation rate $\left(P_{\mathrm{j}}\right.$ tap) modulating the much faster fluctuations of the coronal dissipation rate $\left(P_{\mathrm{x}}\right.$ tap), and therefore a rms-flux correlation is expected. 


\section{Conclusions}

In the last decade, a relatively clear picture of the phenomenology of accretion and ejection in black hole X-ray binaries has emerged. However, from a theoretical perspective many of the observed properties are yet to be understood. The geometry of the accretion flow in the hard state is still debated (ADC versus hot accretion disc). The fundamental physical connection between the hot comptonising plasma and the compact jet of the LHS remains unclear. Moreover we still have no really satisfactory model for the triggering of state transitions and the hysteresis of the spectral evolution during outbursts of transients. Finally as it is suspected for XTE J1118+480, many of the characteristics of the fast X-ray variability of accreting black holes could be associated to some form of disc jet coupling and remain essentially mysterious. The observed timing properties seem to favours models in which the source of photons is close to the black hole, but the tempo of the variability is dictated by a clock located much farther away.

\section{References}

[1] A. A. Zdziarski \& M. Gierliński, Radiative Processes, Spectral States and Variability of Black-Hole Binaries, Progress of Theoretical Physics Supplement, 155, 99-119, (2004).

[2] A.A. Zdziarski, J. Poutanen, W. S. Paciesas, \& L. Wen, Understanding the Long-Term Spectral Variability of Cygnus X-1 with Burst and Transient Source Experiment and All-Sky Monitor Observations,ApJ, 578, 357-373, (2002).

[3] J. Malzac, P. O. Petrucci, E. Jourdain, M. Cadolle Bel, P. Sizun, G. Pooley, C. Cabanac, S. Chaty, T. Belloni, J. Rodriguez, J. P. Roques, P. Durouchoux, A. Goldwurm, \& P. Laurent, Bimodal spectral variability of Cygnus $X-1$ in an intermediate state, $A \& A, 448,1125-1137,(2006)$.

[4] S. Chaty, C. A. Haswell, J. Malzac, R. I. Hynes, C. R. Shrader, \& W. Cui ,Multiwavelength observations revealing the evolution of the outburst of the black hole XTE J1118+480,MNRAS, 346, 689-703, (2003).

[5] J. Malzac, T. Belloni, H. C. Spruit, \& G. Kanbach, The optical and X-ray flickering of XTE J1118+480, A\&A, 407, 335-345, (2003).

[6] J. E. McClintock \& R. A. Remillard, Black hole binaries, in compact Stellar X-ray sources, Eds. W.H.G. Lewin and M. van der Klis, Cambridge University Press, 157-213, (2006) [ astro-ph/0306213].

[7] T. Belloni, J. Homan, P. Casella, M. van der Klis, E. Nespoli, W. H. G. Lewin, J. M. Miller, \& M. Méndez, The evolution of the timing properties of the black-hole transient GX 339-4 during its

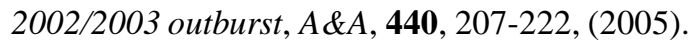

[8] R. Fender, Jets from X-ray binaries, in compact Stellar X-ray sources, Eds. W.H.G. Lewin and M. van der Klis, Cambridge University Press, 381-419, (2006) [astro-ph/0303339].

[9] A.A. Galeev, R. Rosner, \& G. S. Vaiana , Structured coronae of accretion disks, ApJ, 229, 318-326, (1979).

[10] P. Laurent, \& L. Titarchuk, Timing and Spectral Properties of X-Ray Emission from the Converging Flows onto a Black Hole: Monte Carlo Simulations, ApJ, 562, L67-L70, (2001).

[11] A. Niedźwiecki \& A. A. Zdziarski , Bulk motion Comptonization in black hole accretion flows, MNRAS, 365, 606-614, (2006). 
[12] S.L. Shapiro, A. P. Lightman, \& D. M. Eardley, A two-temperature accretion disk model for Cygnus X-1 - Structure and spectrum, ApJ, 204, 187-199, (1976).

[13] J.E. Pringle, Thermal instabilities in accretion discs,MNRAS, 177, 65-71, (1976).

[14] S. Ichimaru, Bimodal behavior of accretion disks - Theory and application to Cygnus X-1 transitions, ApJ, 214, 840-855, (1977).

[15] R. Narayan \& I. Yi , Advection-dominated accretion: A self-similar solution, ApJ, 428, L13-L16, (1994).

[16] M. A. Abramowicz, X.-M. Chen, M. Granath, \& J.-P. Lasota , Advection-dominated Accretion Flows around Kerr Black Holes, ApJ, 471, 762, (1996).

[17] R. D. Blandford \& M. C. Begelman, On the fate of gas accreting at a low rate on to a black hole, MNRAS, 303, L1-L5, (1999).

[18] F. Yuan, Luminous hot accretion discs, MNRAS, 324,119-127, (2001).

[19] G. S. Bisnovatyi-Kogan \& S. I. Blinnikov, A hot corona around a black-hole accretion disk as a model for Cygnus X-1, SvAL, 2, 191-193, (1976).

[20] E. P. T. Liang \& R. H. Price, Accretion disk coronae and Cygnus X-1, ApJ, 218, 247-252, (1977).

[21] K. A. Miller \& J. M. Stone, The Formation and Structure of a Strongly Magnetized Corona above a Weakly Magnetized Accretion Disk, ApJ, 534, 398-419, (2000).

[22] A. Merloni \& A. C. Fabian, Coronal outflow dominated accretion discs: a new possibility for low-luminosity black holes?, MNRAS, 332, 165-175, (2002).

[23] J. Ferreira, Magnetically-driven jets from Keplerian accretion discs, A\&A, 319,340-359, (1997).

[24] R. Svensson \& A. A. Zdziarski, Black hole accretion disks with coronae, ApJ, 436, 599-606, (1994).

[25] F. Haardt \& L. Maraschi , A two-phase model for the X-ray emission from Seyfert galaxies, ApJ, 380, L51-L54, (1991).

[26] F. Haardt \& L. Maraschi , X-ray spectra from two-phase accretion disks, ApJ, 413, 507-517, (1993).

[27] B. E. Stern, M. C. Begelman, M. Sikora, \& R. Svensson, A large-particle Monte Carlo code for simulating non-linear high-energy processes near compact objects, MNRAS,272,291-307, (1995).

[28] J. Poutanen \& R. Svensson, The Two-Phase Pair Corona Model for Active Galactic Nuclei and $X$-Ray Binaries: How to Obtain Exact Solutions, ApJ, 470, 249-(1996).

[29] F. Haardt, L. Maraschi, \& G. Ghisellini, A model for the X-ray and ultraviolet emission from Seyfert galaxies and galactic black holes, ApJ, 432, L95-L99, (1994).

[30] B. E. Stern, J. Poutanen, R. Svensson, M. Sikora, \& M. C. Begelman, On the Geometry of the X-Ray-Emitting Region in Seyfert Galaxies, ApJ, 449, L13, (1995).

[31] J. Malzac, A. M. Dumont, \& M. Mouchet, Full radiative coupling in two-phase models for accreting black holes, A\&A, 430, 761-769, (2005).

[32] A. M. Beloborodov, Plasma Ejection from Magnetic Flares and the X-Ray Spectrum of Cygnus X-1, ApJ, 510, L123-L126, (1999).

[33] J. Malzac, A. M. Beloborodov, \& J. Poutanen, X-ray spectra of accretion discs with dynamic coronae, MNRAS, 326, 417-427, (2001). 
[34] A. A. Zdziarski, P. Lubiński, M. Gilfanov, \& M. Revnivtsev, Correlations between X-ray and radio spectral properties of accreting black holes, MNRAS, 342, 355-372, (2003).

[35] J.M. Miller, J. Homan, D. Steeghs, M. Rupen, R. W. Hunstead, R. Wijnands, P. A. Charles, \& A. C. Fabian, A Long, Hard Look at the Low/Hard State in Accreting Black Holes, ApJ, 653, 525-535, (2006).

[36] E. Gallo, R. P. Fender, \& G. G. Pooley , A universal radio-X-ray correlation in low/hard state black hole binaries, MNRAS, 344, 60-72, (2003).

[37] R. D. Blandford \& A. Konigl, Relativistic jets as compact radio sources, ApJ, 232, 34-48, (1979).

[38] R. M. Hjellming \& K. J. Johnston, Radio emission from conical jets associated with X-ray binaries, ApJ, 328, 600-609, (1988).

[39] A. M.. Stirling, R. E. Spencer, C. J. de la Force, M. A. Garrett, R. P. Fender, \& R. N. Ogley , A relativistic jet from Cygnus X-1 in the low/hard X-ray state, MNRAS, 327, 1273-1278, (2001).

[40] S. Corbel, R. P. Fender, A. K. Tzioumis, M. Nowak, V. McIntyre, P. Durouchoux, \& R. Sood, Coupling of the $X$-ray and radio emission in the black hole candidate and compact jet source GX 339-4, $A \& A$, 359, 251-268, (2000).

[41] S. Markoff, H. Falcke, \& R. Fender, A jet model for the broadband spectrum of XTE J1118+480. Synchrotron emission from radio to X-rays in the Low/Hard spectral state, A\&A, 372, L25-L28, (2001).

[42] S. Markoff, M. A. Nowak, \& J. Wilms, Going with the Flow: Can the Base of Jets Subsume the Role of Compact Accretion Disk Coronae?, ApJ, 635, 1203-1216, (2005).

[43] F. Yuan, A. A. Zdziarski, Y. Xue, \& X.-B. Wu , Modeling the Hard States of XTE J1550-564 during Its 2000 Outburst, ApJ, 659, 541-548, (2007).

[44] E. G. Körding, R. P. Fender, \& S. Migliari , Jet-dominated advective systems: radio and X-ray luminosity dependence on the accretion rate, MNRAS, 369, 1451-1458, (2006).

[45] D. L. Meier, The Association of Jet Production with Geometrically Thick Accretion Flows and Black Hole Rotation, ApJ, 548, L9-L12, (2001).

[46] R. D. Blandford \& D. G. Payne, Hydromagnetic flows from accretion discs and the production of radio jets, MNRAS, 199, 883-903, (1982).

[47] R. D. Blandford \& R. L. Znajek, Electromagnetic extraction of energy from Kerr black holes, MNRAS, 179, 433-456, (1977).

[48] M. Livio, G. I. Ogilvie, \& J. E. Pringle, Extracting Energy from Black Holes: The Relative Importance of the Blandford-Znajek Mechanism, ApJ, 512,100-104, (1999).

[49] Y. Tanaka \& N. Shibazaki , X-ray Novae, ARA\&A, 34, 607-644, (1996).

[50] J. Ferreira, P.-O. Petrucci, G. Henri, L. Saugé, \& G. Pelletier, A unified accretion-ejection paradigm for black hole $X$-ray binaries. I. The dynamical constituents, A\&A, 447, 813-825, (2006).

[51] T.J. Maccarone, Do X-ray binary spectral state transition luminosities vary?, A\&A, 409, 697-706, (2003).

[52] H. C. Spruit \& D. A. Uzdensky, Magnetic Flux Captured by an Accretion Disk, ApJ, 629, 960-968, (2005).

[53] E. Meyer-Hofmeister, B. F. Liu, \& F. Meyer, Hysteresis in spectral state transitions - a challenge for theoretical modeling, A\&A, 432, 181-187, (2005). 
[54] A. Różańska \& B. Czerny, Two-phase radiative/conductive equilibrium in active galactic nuclei and galactic black holes, MNRAS, 316, 473-478, (2000).

[55] M. Mayer \& J. E. Pringle, Time-dependent models of two-phase accretion discs around black holes, MNRAS, 376, 435-456, (2007).

[56] van der Klis, M., A review of rapid X-ray variability in X-ray binaries, in compact Stellar X-ray sources, Eds. W.H.G. Lewin and M. van der Klis, Cambridge University Press, 39-112, [ astro-ph/0410551](2006).

[57] S. Miyamoto \& S. Kitamoto, X-ray time variations from Cygnus X-1 and implications for the accretion process, Natur, 342, 773 (1989).

[58] D. Kazanas, X.-M. Hua, \& L. Titarchuk, Temporal and Spectral Properties of Comptonized Radiation and Its Applications, ApJ, 480, 735, (1997).

[59] J. Malzac \& E. Jourdain, Temporal properties of flares in accretion disk coronae, A\&A, 359, 843-854, (2000).

[60] M. Böttcher, Coronal Cooling and Its Signatures in the Rapid Aperiodic Variability of Galactic Black Hole Candidates, ApJ, 553, 960-967, (2001).

[61] J. C. Lochner, J. H. Swank, \& A. E. Szymkowiak, Shot model parameters for Cygnus X-1 through phase portrait fitting, ApJ, 376, 295-311, (1991).

[62] M. A. Nowak, \& R. V. Wagoner, Accretion disc turbulence and the X-ray power spectra of black hole high states, MNRAS, 274, 37-47, (1995).

[63] J. Poutanen \& A. C. Fabian, Spectral evolution of magnetic flares and time lags in accreting black hole sources, MNRAS, 306, L31-L37, (1999).

[64] J. F. Hawley \& J. H. Krolik, Global MHD Simulation of the Inner Accretion Disk in a Pseudo-Newtonian Potential, ApJ, 548, 348-367, (2001).

[65] P. Uttley, I. M. McHardy, \& S. Vaughan, Non-linear X-ray variability in X-ray binaries and active galaxies, MNRAS, 359, 345-362, (2005).

[66] Y. E. Lyubarskii, Flicker noise in accretion discs, MNRAS, 292, 679, (1997).

[67] O.. Kotov, E. Churazov, \& M. Gilfanov, On the X-ray time-lags in the black hole candidates, MNRAS, 327, 799-807, (2001).

[68] A.R. King, J. E. Pringle, R. G. West, \& M. Livio, Variability in black hole accretion discs, MNRAS, 348, 111-122, (2004).

[69] M. Mayer \& J. E. Pringle, Variability of black hole accretion discs: the cool, thermal disc component, MNRAS, 368, 379-396, (2006).

[70] E. Churazov, M. Gilfanov, \& M. Revnivtsev, Soft state of Cygnus X-1: stable disc and unstable corona, MNRAS, 321, 759-766, (2001).

[71] G. Kanbach, C. Straubmeier, H. C. Spruit, \& T. Belloni, Correlated fast X-ray and optical variability in the black-hole candidate XTE J1118+480, Natur, 414, 180-182, (2001).

[72] J. Malzac, A. Merloni, \& A. C. Fabian, Jet-disc coupling through a common energy reservoir in the black hole XTE J1118+480, MNRAS, 351, 253-264, (2004). 